\title{
Update on the emergency medical treatment of anaphylactic reactions for first medical responders and for community nurses
}

\author{
Project Team of The Resuscitation Council (UK)
}

Consensus guidelines on the emergency treatment of anaphylactic reactions were published in 1999 in the Fournal of Accident and Emergency Medicine, ${ }^{1}$ and in Resuscitation. ${ }^{2}$ These guidelines were intended for first medical responders, and more specifically those without expert knowledge or specialist interest. In general they have been well received.

After the publications, we received a large number of letters from community nurses. They explained that adherence to the new guidelines was difficult in their situation because they did not have access to the drugs that were mentioned, apart from adrenaline (epinephrine). Moreover, many were troubled by the recommendations for children's doses that included a suggestion of using the twofold dilution for the lowest dose.

The British National Formulary also offers advice on anaphylaxis, and there was some concern on all sides that the doses of adrenaline (epinephrine) were different in the British National Formulary from those given in the consensus guidelines.

Correspondence to: Professor D Chamberlain, 25 Woodland Drive, Hove, East Sussex BN3 6DH, UK (chambda@pavilion.co.uk)

Accepted for publication 11 May 2001

Figure 1 Anaphylactic reactions: treatment for adults by first medical responders. ${ }^{\star} A n$ inhaled $\beta$ agonist such as salbutamol may be used as an adjunctive measure if bronchospasm is severe and does not respond rapidly to other treatment. fIf profound shock judged immediately life threatening give CPR/ALS if necessary. Consider slow intravenous (IV) adrenaline (epinephrine) 1:10 000 solution. This is hazardous and is

recommended only for an experienced practitioner who can also obtain IV access without delay. Note the different strength of adrenaline (epinephrine) that may be required for $I V$ use. $\neq$ If adults are treated with an EpiPen, the $300 \mu g$ will usually be sufficient. A second dose may be required. Half doses of adrenaline (epinephrine) may be safer for patients on amitriptyline, imipramine, or $\beta$ blocker. $\$$ A crystalloid may be safer than a colloid.
In order to resolve both of these problems, a meeting was held between representatives of the Project Team, of the British National Formulary, and of the Department of Health (representing the interests of community nurses).

The meeting was wholly successful, and as a result we have made some very small changes

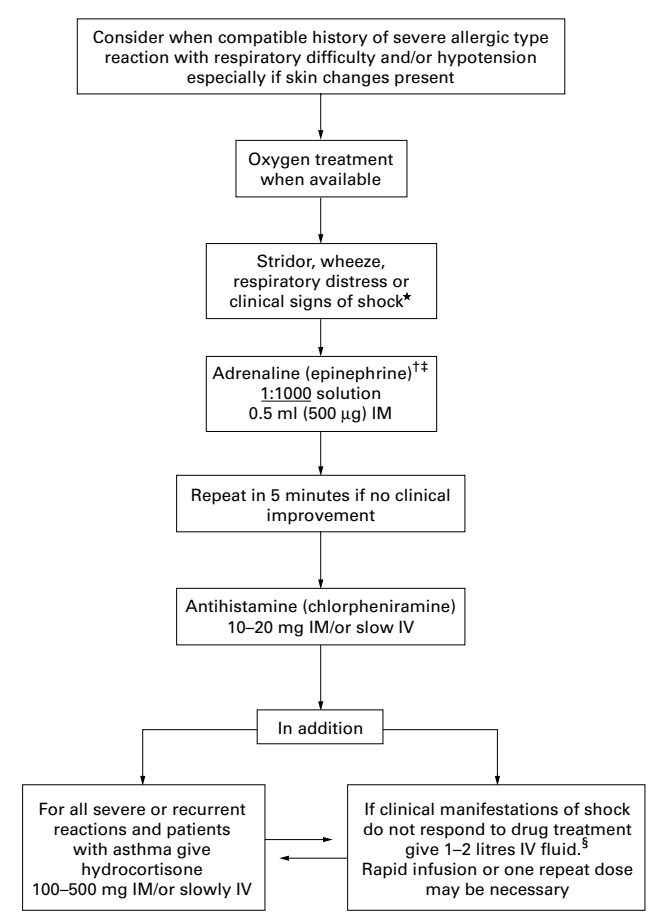

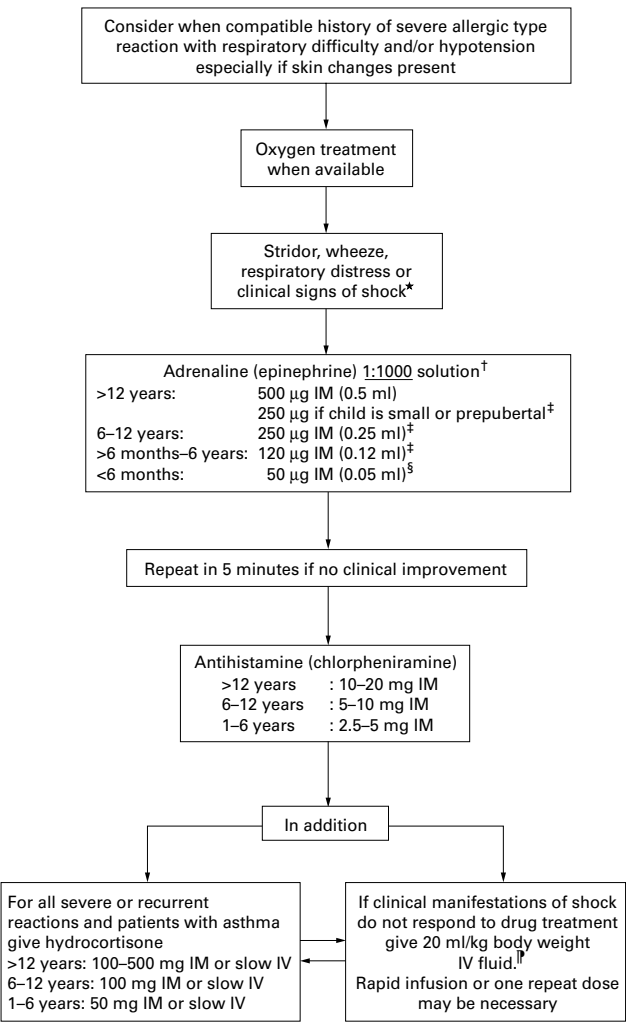

Figure 2 Anaphylactic reactions: treatment for children by first medical responders. ${ }^{*}$ An inhaled $\beta_{2}$ agonist such as salbutamol may be used as an adjunctive measure if bronchospasm is severe and does not respond rapidly to other treatment. + For profound shock judged immediately life threatening give CPR/ALS if necessary. Consider slow intravenous (IV) adrenaline (epinephrine) 1:10 000 solution. This is hazardous and is recommended only for an experienced practitioner who can also obtain IV access without delay. Note the different strength of adrenaline (epinephrine) that may be required for IV use. $\neq$ For children who have been prescribed EpiPen, $150 \mu \mathrm{g}$ can be given instead of $120 \mu \mathrm{g}$, and $300 \mu \mathrm{g}$ can be given instead of $250 \mu \mathrm{g}$ or $500 \mu \mathrm{g}$. SAbsolute accuracy of this small dose is not essential. $\uparrow$ A crystalloid may be safer than a colloid.

in the first medical responder document to the doses of adrenaline (epinephrine) for children, and we have also taken the opportunity of modifying the age brackets to bring them closer into line with the recommendations from the Royal College of Paediatrics and Child Health. ${ }^{3}$ We have also prepared modified simpler algorithms for community nurses that do not include any drugs apart from adrenaline (epinephrine).

Figures 1 and 2 show the first medical responder guidelines for adults and for children incorporating the very minor changes that have been made. The changes are as follows: 


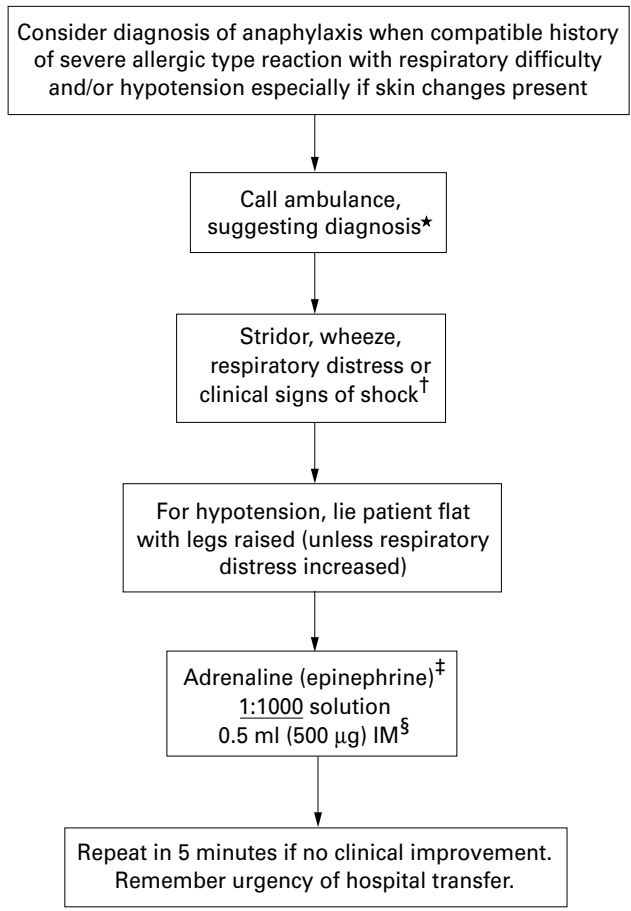

Figure 3 Anaphylactic reactions: treatment for adults in the community. *Ambulance will be equipped with oxygen, salbutamol, and fluids that may be used as adjunctive therapy. IIf profound shock judged to be immediately life threatening give CPR/ALS if necessary. $\neq$ Half doses of adrenaline (epinephrine) may be safer for patients on amitriptyline, imipramine, or $\beta$ blocker. If adults are treated with an EpiPen, the $300 \mu \mathrm{g}$ will usually be sufficient. A second dose may be required, but this should be considered ONLY if the patient's condition continues to deteriorate five minutes after the first dose. NB Remember the urgency of hospital transfer.

1 In the adult algorithm footnote $\ddagger$ offers advice in relation to adults who are treated with an Epipen. This is an increasingly common practice. A caution has been added in relation to tricyclics and $\beta$ blockers.

2 In the children's algorithm, the age brackets are now $<6$ months, $>6$ months to 6 years, 6 years to 12 years, and $>12$ years.

3 The dose of adrenaline (epinephrine) for young children has been changed slightly from $125 \mu \mathrm{g}$ to $120 \mu \mathrm{g}$, which is believed to be more realistic.

4 Likewise the smallest dose for infants is now $50 \mu \mathrm{g}$ instead of $62.5 \mu \mathrm{g}$ without any advice for twofold dilution.

5 Identical doses of adrenaline (epinephrine) have been recommended for use by community nurses.

These changes are relatively inconsequential, but the new doses are preferred by community nurses and we wish to achieve consistency of recommendations. The simplified algorithms for community nurses are shown in figures 3 and 4 .

The new doses are now compatible with the advice to be offered in the British National Formulary and identical to those that will appear in the new version of the Green Book to be published by the Department of Health in relation to immunisation and vaccination.

The Project Team of the Resuscitation Council (UK) is pleased that the objective of

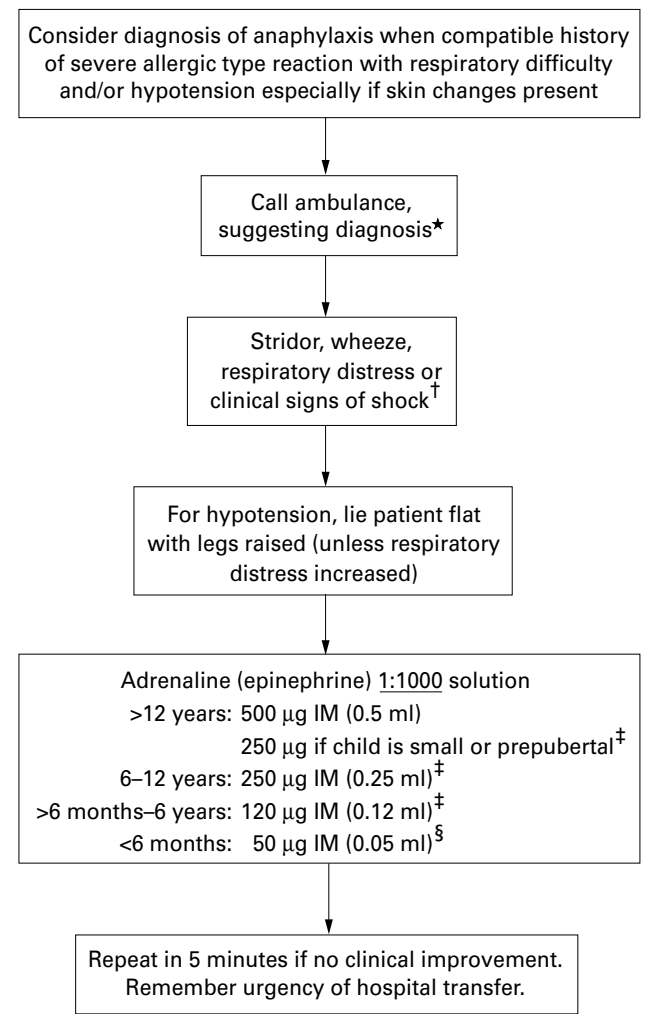

Figure 4 Anaphylactic reactions: treatment for children in the community. ${ }^{*}$ Ambulance will be equipped with oxygen, salbutamol, and fluids that may be used as adjunctive therapy. IIf profound shock judged to be immediately life threatening give CPR/ALS if necessary. $\neq$ For children who have been prescribed EpiPen, $150 \mu \mathrm{g}$ can be given instead of $120 \mu \mathrm{g}$, and $300 \mu \mathrm{g}$ can be given instead of $250 \mu \mathrm{g}$ or $500 \mu \mathrm{g}$. SAbsolute accuracy of this small dose is not essential.

uniform guidelines for first medical responders (and now for community nurses as well) has been realised. The consensus guidelines are not intended to replace guidelines for specialist groups in hospitals. ${ }^{4-8}$

\section{Members of Project Team:}

Professor Douglas Chamberlain (Chairman), Dr Barbara Phillips (also for the Royal College of Paediatrics and Child Health), Dr Judith Fisher (also for the Royal College of General Practitioners), Dr Michael Ward (also for The Association of Anaesthetists). Coopted: Dr Andrew Cant (for the Royal College of Paediatrics and Child Health), Dr Peter Dawson (for Royal College of Radiologists), Dr Pamela Ewan, Mrs Angela Fritz (for the Anaphylaxis Campaign), Dr Gideon Lack, Professor Tak Lee (for the British Society for Allergy and Clinical Immunology), Dr John Martin (for the British National Formulary), Dr Richard Pumphrey (for the Royal College of Pathologists), Dr George Rylance (for the Royal College of Paediatrics and Child Health), Mr Howard Sherriff (for British Association of Emergency Medicine), Professor David Warrell (for the Royal College of Physicians), Dr David Salisbury (Principal Medical Officer, Dept of Health), Mrs Marilyn Eveleigh (Nurse Adviser in Primary Care and Public Health, East Sussex Area Health Authority).

This paper has also appeared in Resuscitation 2001:48:241-3.

1 Project Team of the Resuscitation Council (UK). Emergency medical treatment of anaphylactic reactions. F Accid Emerg Med 1999;16:243-8.

2 Project Team of the Resuscitation Council (UK). The emergency medical treatment of anaphylactic reactions. Resuscitation 1999;41:93-9.

3 Royal College of Paediatrics and Child Health. Medicines for children. London: Royal College of Paediatrics and Child Health, 1999:viii. 
4 Association of Anaesthetists of Great Britain and Ireland and The British Society of Allergy and Clinical Immunology. Suspected anaphylactic reactions asso
sia. Revised edition. London: 1995 .

5 Board of Faculty of Clinical Radiology, Royal College of Radiologists. Advice on the management of reactions to intravenous contrast media. London: Royal College of Radiologists, 1996
6 Gavalas M, Sadana A, Metcalf S. Guidelines for the management of anaphylaxis in the emergency department. f Accid Emerg Med 1998;15:96-8.

7 Gavalas M, Walford C, Sadana A, et al. Medical treatment of anaphylaxis. [Letter]. F Accid Emerg Med 2000;17:152.

8 Chamberlain D for the anaphylaxis project team. Emergency treatment of anaphylactic reactions. [Authors' reply].

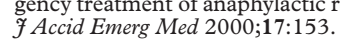

